\title{
Simulation par la Méthode des Eléments Finis du Comportement Vibratoire d'un Rotor
}

\author{
Simulation by the Finite Element Method of the Vibratory behaviour of a
}

\section{Rotor}

\author{
Trésor Kanyiki ${ }^{1}$ \\ ${ }^{1}$ Département d'électromécanique, Faculté Polytechnique, Université de Lubumbashi, République Démocratique du \\ Congo, tresorkanyiki@gmail.com
}

\begin{abstract}
RÉSUMÉ. Cet article est consacré à l'étude du comportement vibratoire d'un rotor. L'étude présente un ensemble des techniques scientifiques sur la modélisation et simulation des vibrations d'un rotor d'une turbomachine entrainée en torsion. Cette étude permet de comprendre à partir de l'étude dynamique l'origine des bruits et des vibrations d'un rotor ; l'étude permet à partir du logiciel Rotorinsa de mettre en exergue les effets du balourd sur un rotor. Pour élaborer les équations du mouvement vibratoire du rotor, nous avons utilisé l'approche énergétique de Lagrange. Pour y arriver, un modèle à deux roues portées par un arbre supporté par deux paliers hydrodynamiques est choisi conformément aux caractéristiques du rotor étudié. Chaque élément du rotor est défini par son élément fini. La résolution analytique des équations différentielles qui caractérisent le comportement vibratoire du rotor est ardue manuellement.

L'approche numérique faisant usage de la méthode des éléments finis, programmée sur le logiciel Rotorinsa a permis d'effectuer l'analyse dynamique du rotor.

ABSTRACT. This article is dedicated to the study of the vibratory behavior of a rotor. The study presents a set of scientific techniques on modeling and simulation of the vibrations of a rotor of a turbomachinery driven in torsion. This study helps to understand from the dynamic study the origin of the noise and vibration of a rotor; the study allows from the Rotorinsa software to highlight the effects of unbalance on a rotor. To develop the equations of the vibratory motion of the rotor, we used Lagrange's energy approach. To succeed, a two wheeled model carried by a tree supported by two hydrodynamic bearings is chosen according to the characteristics of the studied rotor. Each element of the rotor is defined by its finite element. The analytical resolution of the differential equations that characterize the vibratory behavior of the rotor is difficult manually. The numerical approach using the finite element method, programmed on Rotorinsa software allowed to perform dynamic rotor analysis.
\end{abstract}

MOTS-CLÉS. Rotor, équation de Lagrange, modélisation, vibration, éléments finis, balourd.

KEYWORDS. Rotor, Lagrange's equation, modelization, vibration, finite element, oaf.

\section{Introduction}

Dans le secteur industriel tel que : la mécanique automobile ou industrie mécanique, l'industrie nucléaire, la mécanique aéronautique, ... il est question de transformer une forme d'énergie dans une autre forme d'énergie par l'intermédiaire d'un organe mécanique. La machine tournante est constituée d'un rotor et d'un stator. Actuellement, l'industrie moderne ne peut se passer des machines tournantes et font l'objet d'un système d'ingénierie concurrentielle [BRA 10] et [CHE 08]. Dès la phase de la conception et de fabrication, ces machines doivent répondre à un cahier de charge bien défini ; cela a conduit à l'optimisation des performances et le rendement des machines tournantes. La tendance actuelle vise à réduire les jeux entre le rotor et le stator, à réduire la masse totale de la machine tournante tout en augmentant sa vitesse de rotation, à accroitre sa durée de vie par une amélioration de sa fiabilité par des techniques de maintenance [STE 96].

Par ailleurs, un système oscille toujours lorsqu'il est en mouvement relatif. Il effectue un mouvement de va et vient autour de la position d'équilibre. Dans certaines conditions, les efforts générés par les machines tournantes au cours de leurs fonctionnements peuvent devenir importantes, et provoquer la mise hors service prématurée de l'ensemble du système. En pratique, l'arbre est l'élément le plus sollicité par les efforts dynamiques générés. Les causes des déformations de l'arbre sont les 
suivantes : les vibrations longitudinales (ou axiales), les vibrations de rotation causées par la torsion et les vibrations transversales (ou latérales) [GHA 14] et [LAL 96].

Les phénomènes vibratoires sont caractérisés par un mode propre, valeurs propres, vecteurs propres, fréquences propres. Ils sont appelés caractéristiques modales. La sûreté de fonctionnement des machines tournantes exige que les fréquences de source excitatrices ne coïncident pas avec les fréquences propres de la machine. Sinon, la résonance se produit, il conduit à la mise hors service prématuré de la machine tournante. La dynamique du rotor permet de cerner, connaitre et de maitriser les phénomènes cités ci-haut. Sur une machine tournante, les phénomènes vibratoires peuvent conduire à l'instabilité ou à l'endommagement du système, caractérisé par la rupture ou la fatigue, frottement entre rotor et stator, endommagement des paliers, les bruits dus au fonctionnement du rotor. La vitesse critique accélère ce phénomène. L'étude dynamique des machines tournantes s'avère indispensable.

Les machines tournantes tiennent le monopole de la production dans les secteurs industriels. Lorsque les vibrations et les bruits apparaissent sur une machines tournantes, il est important de connaitre les causes et de prédire les conséquences, de faire l'étude dynamique afin de caractériser les anomalies et effectuer l'étude du comportement dynamique. Cet article se fixe pour objectif de connaitre l'influence d'un balourd sur un rotor dont les vibrations et les bruits deviennent excessifs ; sachant que l'alignement du rotor, les conditions de fonctionnement sont bonnes.

\section{Description des éléments d'un rotor}

Une turbomachine est un dispositif qui donne ou retire l'énergie à un fluide à partir de l'action dynamique de l'élément rotation autour d'un axe appelé rotor [REG 05]. Les turbomachines sont rencontrées dans les secteurs industriels nécessitant un transfert d'énergie.

Dans l'industrie électrique, par usage des éoliennes (énergie du vent), les turbines à gaz ou à vapeur, les hydroliennes ou encore dans les centrales hydroélectriques. Dans les grandes industries, les turbomachines jouent un rôle fondamental, les compresseurs centrifuges par exemple pour alimenter en air les unités qui transforment les produits ; les ventilateurs pour aération ou aspiration des flammes etc, les turbopompes sont utilisées dans la distribution de l'eau ou en exhaure, les turbocompresseurs, ... dans le secteur du transport, on utilise les ventilateurs dans le système d'aération, en aéronautique, les turbosoufflantes et turbo aérateurs assurent la propulsion des engins, les turbopompes alimentent les moteurs cryogéniques dans les engins aérospatiaux, etc. peu importe la forme ou l'usage d'une turbomachine, le rotor qui est la partie motrice de la machine est constitué de 4 éléments : arbre sur lequel on retrouve une série de roues; les roues sont modélisées par un tronc de cône ou par un disque de forme cylindrique. Sur la roue, sont fixée des pales qui peuvent être modélisées par des poutres encastrées. On retrouve aussi le balourd. Enfin les paliers sur lesquels reposent l'ensemble arbre-rouebalourd [REG 05].

\subsection{Définition du rotor}

Le rotor d'une turbomachine est constitué de l'arbre, le disque, le balourd, les paliers et l'aube [JEN 05]. Ces éléments sont visibles à la figure 1 . 


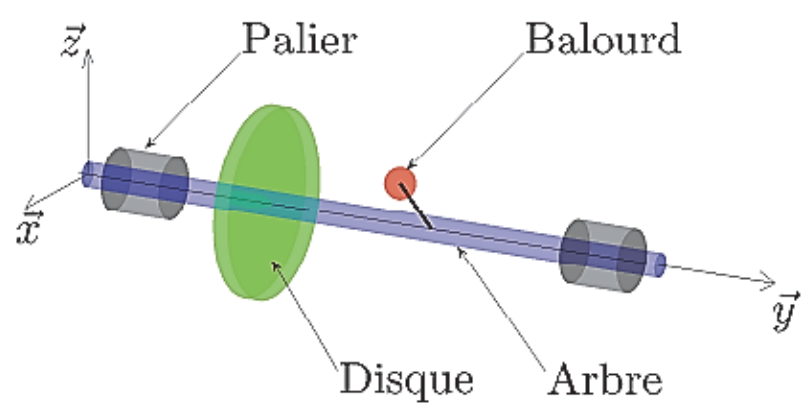

Figure 1. Description du rotor [JEN 05]

\subsubsection{Disque}

La roue peut être modélisée par un disque, il peut être déformable ou rigide. Une roue peut être représentée géométriquement sous forme cylindrique; mais dans l'analyse dynamique, cette forme s'avère limitée. La roue est généralement représentée en forme de tronc de cône. Pour une roue rigide, seule son énergie cinétique intervient dans l'analyse dynamique [REG 05]. Sur la figure 2 effectuée sur solidwork, nous avons une roue d'une turbine Francis.

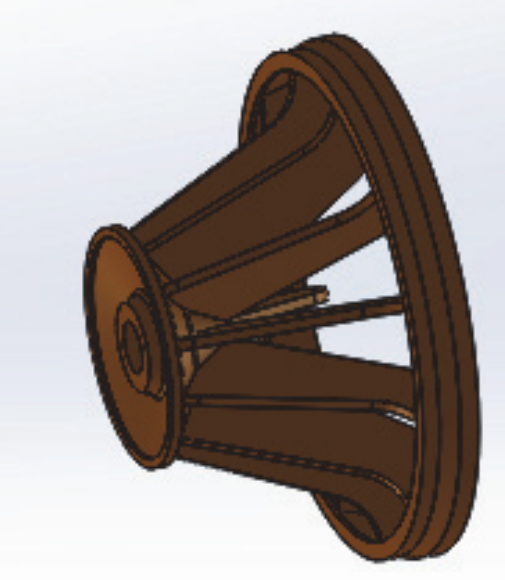

Figure 2. Roue de la turbine Francis

\subsubsection{Arbre}

C'est un organe qui porte les roués. Il peut être rigide ou flexible, ces propriétés dépendent de son matériau. L'arbre est considéré déformable dans l'analyse dynamique, cela permet de tenir compte des effets des déformations [REG 05]. Géométriquement, la section de l'arbre peut être constante ou variable. L'étude en flexion devient complexe pour l'arbre à section variable du fait que le mouvement est décrit par les équations différentielles linéaires à coefficients variables dont les solutions sont les fonctions de Bessel [REG 05].

\subsubsection{Aube}

L'aube est un organe fixé sur la roue, sa modélisation se fait par une poutre encastrée à l'une des extrémités. Elle peut être flexible ou rigide. Pour une étude du comportement dynamique des roues aubagées, elles sont considérés flexibles [JEN 05].

\subsubsection{Palier}

Le palier est aussi appelé support, il peut être flexible (isotrope ou anisotrope) ou rigide. Le palier constitue un système de dissipation ou non lorsque la flexibilité est anisotrope. L'arbre du rotor loge 
dans un carter, la surface fonctionnelle du carter est séparée de celle de palier par un matériau antifriction et d'un film d'huile. L'huile ou la graisse assure la lubrification afin de réduire la friction entre les deux surfaces [GOJ 99] et [TAL 07].

On parle des paliers hydrodynamiques (avec fluide liquide) lorsque le film de fluide est généré par le mouvement relatif des deux surfaces. Ils sont largement utilisés en turbomachine, leur fonctionnement est illustré à la figure 3 : en effet, la rotation de l'arbre génère une raideur à l'intérieur du palier et un amortissement visqueux dont les caractéristiques dépendent de la charge (poids propre et autres forces) appliquée sur les paliers par le rotor, la géométrie du palier, de la vitesse de rotation et le rapport L/D (largeur/diamètre), des variations de sa température et de la viscosité de l'huile utilisée. Pour calculer ces paramètres qui sont des fonctions non linéaires des charges appliquées aux paliers. On modélise l'équilibre des forces de pression du film d'huile à l'aide de l'équation de Reynolds, dans les directions horizontales et verticales [GOJ 99] et [TAL 07].

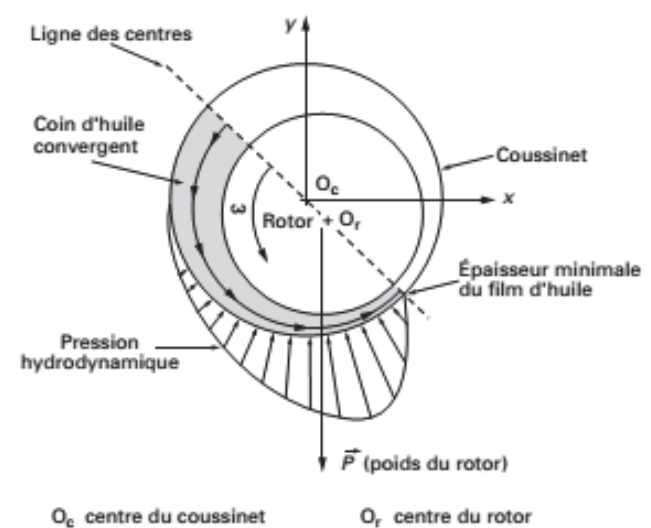

Figure 3. Fonctionnement d'un palier hydrodynamique [GOJ 99]

D'autres types des paliers sont: paliers aérostatiques (film fluide gazeux), les paliers aérodynamiques (film fluide gazeux), les paliers hydrostatiques (le film fluide liquide est injecté sous haute pression), lorsqu'on insère les éléments roulant (galets, billes, aiguilles) entre les surfaces, on parle de paliers à roulements [GOJ 99].

\subsubsection{Balourd}

Le balourd est toute masse excentrée $m_{b}$ d'un rotor. Il est localisé en une distance $d$ du centre de géométrique de l'arbre comme montré à la figure 4 [REG 05].

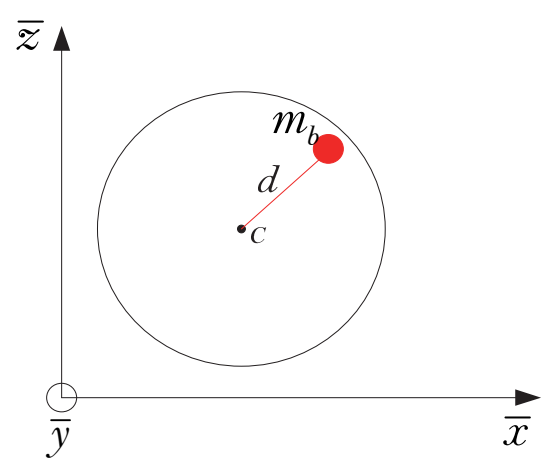

Figure 4. Elément de balourd

Les balourds ont différentes origines. On parle souvent des inhomogénéités du matériau, mais il existe d'autres causes : 


\subsubsection{Géométrie des pièces tournantes}

La distribution de balourd d'un rotor dépend de la position du centre de masse de chaque tronçons par rapport à l'axe de rotation, qui est diffèrent de l'axe géométrique qui passe par les centres des paliers. Pour des rotors monoblocs constitués des roues usinées dans la masse, on observe également une modification de la position des centres de gravité due aux écarts d'usinages [PUG 03].

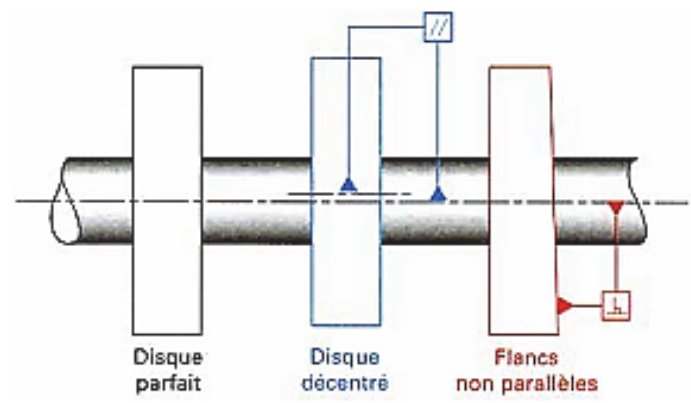

Figure 5. Ecart d'usinage (excentricité et parallélisme) cause de balourd [PUG 03]

Par exemple, la distribution naturelle des ailettes de turbine donne un balourd qui dépend de leurs positions relatives de montage [PUG 03].

\subsubsection{Assemblage des pièces}

Les pièces assemblées sur un arbre en rotation déplacent naturellement leurs centres de masse. C'est le cas des turbines, moteurs électriques, ... il est crucial de veiller sur le procédé d'assemblage pouvant faire de deux pièces assemblées un bloc balourdé [GOJ 99].

\subsubsection{Conditions de service}

On peut citer les causes dues à la variation de la température au voisinage de la machine en service. On peut aussi parler de la relaxation des contraintes, l'usure non axisymétrique etc. susceptible d'apporter des balourds qui peuvent évoluer avec le temps [BRA 10].

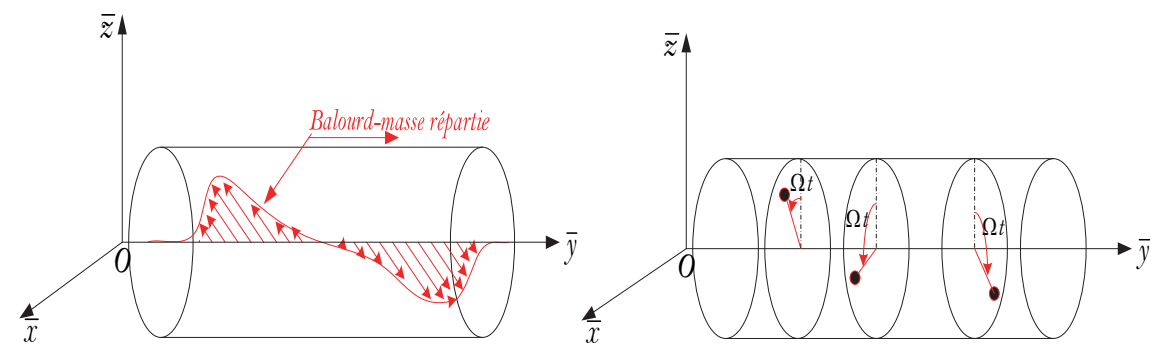

Figure 6. Modèle discrétisé de balourd dans le rotor (à droite) et Profil de distribution de balourd dans le rotor (à gauche) [BRA 10] et [GHA 14]

Généralement, le balourd se répartit de façon aléatoire et de façon continue (figure 6). Pour modéliser de manière simple, on le décompose en plusieurs masses concentriques situées dans différents plans (figure 6). Ces masses concentriques comparativement au balourd continu, sont supposées avoir un même effet sur le comportement dynamique du rotor [LAL 96]. La norme DIN ISO 1940-1 défini les masses de balourd admissibles au regard de la masse du corps à équilibrer en fonction de la vitesse de rotation maximale du corps et en fonction des divers niveaux de qualité notée G [LAL 96].

Dans cette norme, le groupe G6.3 pour les rotors de ventilateurs, de soufflerie et de roues d'avions, le G16 correspond aux rotors d'entrainement non critique, le G2.5 pour les rotors d'entrainement 
machine outils et turbines, le G1 pour les pièces d'entrainement de tours, les platines de tourne disque et le G0.4 pour les gyroscopes et les rotors de tour de précision [LAL 96].

\subsubsection{Types de rotors}

Du pont de vue structure mécanique et forme géométrique, les rotors des turbomachines sont distincts. Du point de vue forme géométrique, on distingue des rotors à arbre long (figure 7.a) : les dimensions de la roue sont petites que celle de l'arbre. Ce type de rotor modélise physiquement certaines turbomachines comme les turbines; les disques rotor (figure 7.b) dont les dimensions de l'arbre sont petits par rapport à celles de l'arbre. Les dispositifs tels que les ventilateurs, des micro turbines, le disque dur des unités centrales ou ordinateurs portables, la meuleuse, etc... sont modélisés par ce type de rotor lors de l'étude du comportement vibratoire; les rotors à ailes ou à aubages (figure 7.c), ils sont utilisés pour modéliser les turbines Pelton, les rotors verticaux des hélicoptères, les éoliennes, les soufflantes industrielles, ou toute autre structure aubagée ; les rotors libres (figure 7.d), n'ont pas des supports [BRA 10].
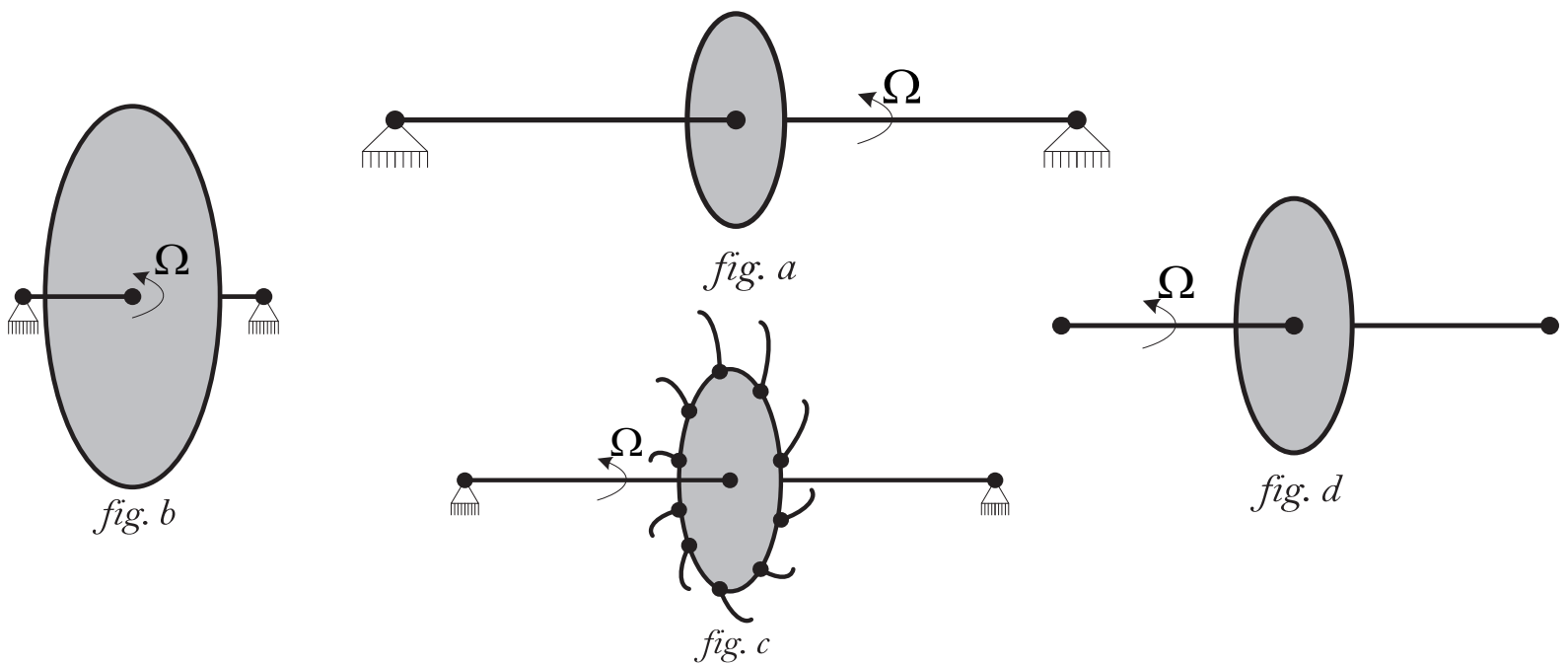

Figure 7. Rotor à arbre long (a), Rotor type disque arbre (b), Rotor à aubes (c), Rotor libre (d).

Selon la structure, on distingue : les rotors flexibles, l'arbre tourne à des vitesses supérieures à la vitesse critique et les déformations sont prises en compte ; les rotors rigides, les éléments de rotors ne se déforment pas et toute la charge dynamique se transmet dans les paliers; les rotors de Jeffcott (figure 8), il est bien équilibré, les effets gyroscopiques sont négligeables, ce rotor consiste à une masse attachée au centre de la section élastique d'un arbre de masse négligeable [BRA 10].

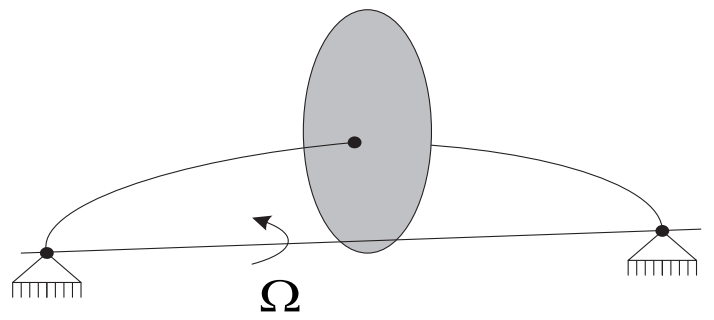

Figure 8. Rotor de Jeffcott.

\subsection{Présentation du rotor étudié}

Le rotor faisant l'objet d'étude dans cet article est illustré sur la figure 2 . Il est présenté à la figure 1 la turbine hydraulique de type Francis L14 qui se trouve à la centrale hydroélectrique de la Lubilanji I 
en République Démocratique du Congo. Cette centrale alimente la province du Kasaï oriental (République Démocratique du Congo) en énergie électrique. Au cours de nos visites à la ligne d'arbre de la centrale hydroélectrique, nous avons perçu des bruits et des vibrations au sol, cet artticle se pour objectif de comprendre à partir de l'analyse dynamique du rotor, l'origine des bruits et des vibrations.

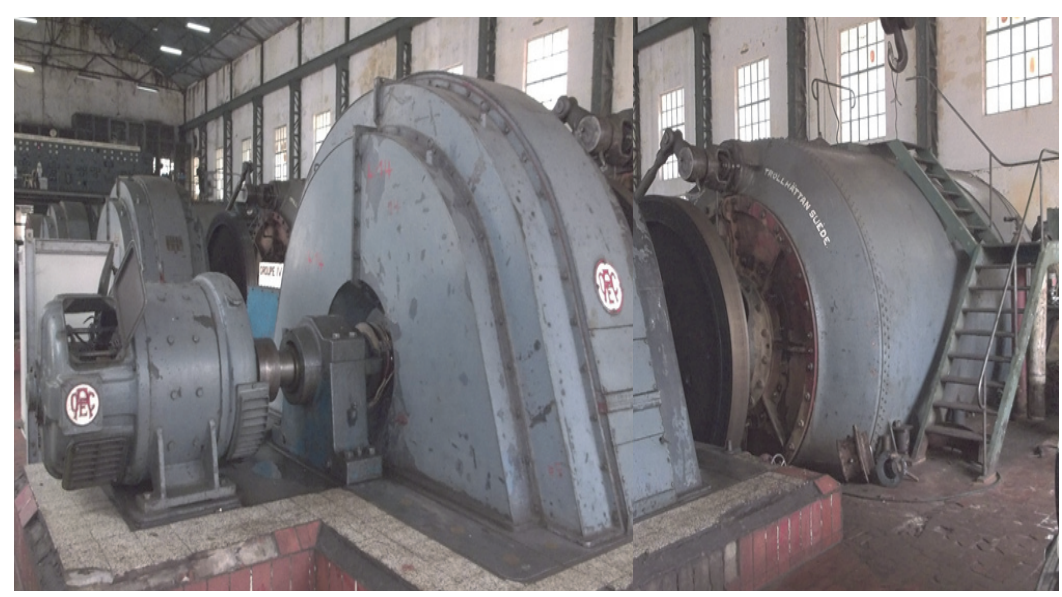

Figure 9. Vue panoramique de la ligne d'arbre (Photo in situ)

Elle a une capacité installée de $7200 \mathrm{kw}$, on retrouve 2 turbines hydraulique de type Francis montées sur ligne d'arbre.

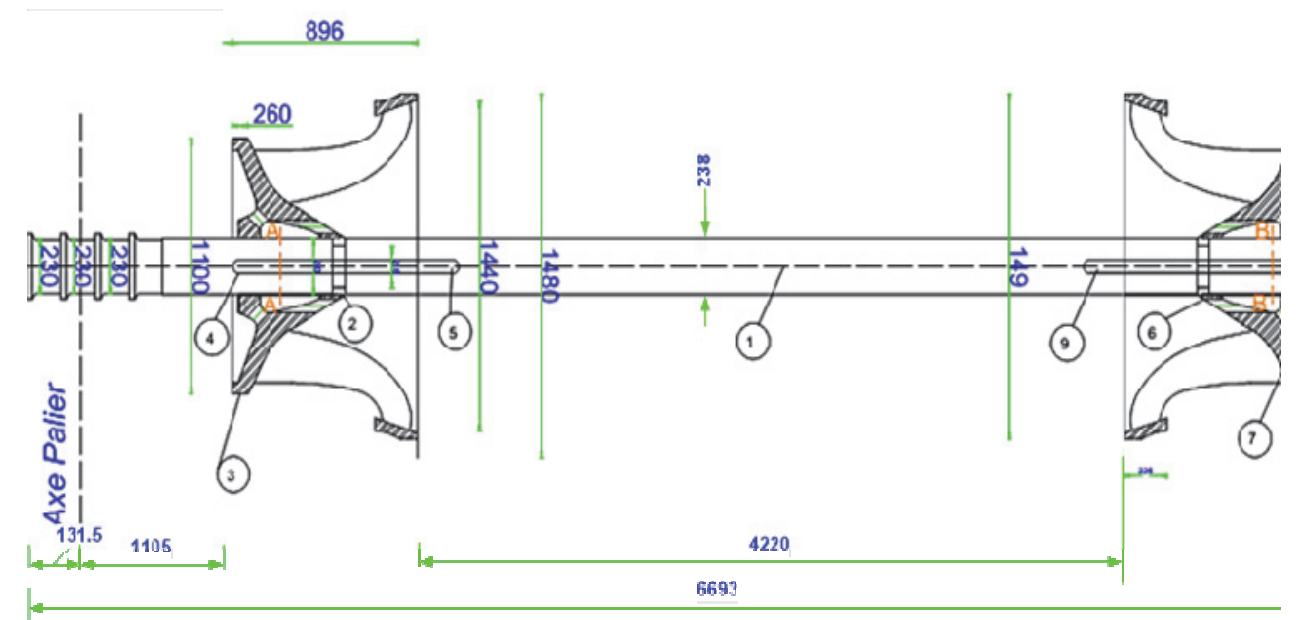

Figure 10. Rotor de la turbine hydraulique L14 de la centrale hydroélectrique de Lubilanji

Sur la figure 2., nous avons : 1. Arbre (ou axe), 2. Anneau buté (coté aval), 3. Roue à aubes (coté aval), 4. Cale, 5. Contre cale, 6. Anneau buté (coté amont), 7. Roue à aubes (coté amont), 8.Cale et 9. Contre cale.

\subsubsection{Caractéristiques géométriques et mécaniques du rotor}

Cette partie présente les caractéristiques géométriques et dynamiques de l'arbre. Ces données sont prises sur le site de la centrale Lubilanji. 


\begin{tabular}{|c|c|c|c|c|}
\hline \multirow[t]{2}{*}{ Elements } & \multicolumn{4}{|c|}{ Caractéristiques } \\
\hline & \multicolumn{2}{|c|}{ Géométriques } & \multicolumn{2}{|c|}{ Mécaniques } \\
\hline \multirow{7}{*}{ Disque } & Grandeurs & Valeurs & Grandeurs & Valeurs \\
\hline & $\begin{array}{l}\text { Rayon extérieur } \\
\mathrm{R}_{1}\end{array}$ & $645 \mathrm{~mm}$ & Matériaux & Fonte \\
\hline & $\begin{array}{l}\text { Rayon intérieur } \\
\mathrm{R}_{2}\end{array}$ & $238 \mathrm{~mm}$ & $\rho$ & $7300 \mathrm{Kg} / \mathrm{m}^{3}$ \\
\hline & Epaisseur e & $896 \mathrm{~mm}$ & $\mathrm{E}$ & $190000 \mathrm{MPa}$ \\
\hline & \multicolumn{2}{|c|}{ Position des disques } & Masse & \\
\hline & Disque gauche $l_{1}$ & $1105 \mathrm{~mm}$ & disque Ma & $7384.7 \mathrm{Kg}$ \\
\hline & Disque droite $l_{2}$ & $6561.5 \mathrm{~mm}$ & Type & Rigide \\
\hline
\end{tabular}

Tableau 1. Caractéristiques géométrique et mécanique de l'arbre

Les grandeurs géométriques et mécaniques sont utiles lors de la simulation du comportement dynamique de l'arbre. Le tenseur d'inertie de la roue est donnée par :

$$
I_{G}(\text { Roue })=\left(\begin{array}{ccc}
1366,6 & 0 & 0 \\
0 & 1745 & 0 \\
0 & 0 & 1366,6
\end{array}\right) \mathrm{kgm}^{2}
$$

\begin{tabular}{|c|c|c|c|c|}
\hline & Grandeurs & Valeurs & Grandeurs & Valeurs \\
\hline & & & Matériaux & Acier \\
\hline & & & $\rho$ & $7700 \mathrm{Kg} / \mathrm{m}^{3}$ \\
\hline Arbre & $\mathrm{L}$ & $6693 \mathrm{~mm}$ & $\mathrm{E}$ & $\begin{array}{r}210000 \\
\mathrm{MPa}\end{array}$ \\
\hline & & & $\mathrm{M}$ & $3000 \mathrm{Kg}$ \\
\hline & $\mathrm{D}$ & $238 \mathrm{~mm}$ & Type & Flexible \\
\hline Balourd & Excentricité & $\mathrm{e}=645 \mathrm{~mm}$ & $\mathrm{M}_{\mathrm{b}}$ & $30 \mathrm{~g}$ \\
\hline & Grandeurs & Valeurs & Grandeurs & Valeurs \\
\hline & Position palier & & $\mathrm{k}_{\mathrm{A}}$ & $5.410^{6} \mathrm{~N} / \mathrm{m}$ \\
\hline Doliare & Palier gauche & $\begin{array}{c}\mathrm{y}=131.5 \\
\mathrm{~mm}\end{array}$ & $\mathrm{k}_{\mathrm{xx}}$ & $10^{8} \mathrm{~N} / \mathrm{m}$ \\
\hline Iants & Palier droit & $\begin{array}{c}\mathrm{y}=6561.5 \\
\mathrm{~mm}\end{array}$ & $\mathrm{k}_{\mathrm{zz}}$ & $2.410^{8} \mathrm{~N} / \mathrm{m}$ \\
\hline & & & $\begin{array}{l}C_{x x} \\
C_{z z}\end{array}$ & $\begin{array}{l}10^{2} \mathrm{Ns} / \mathrm{m} \\
2.410^{2} \mathrm{Ns} / \mathrm{m}\end{array}$ \\
\hline
\end{tabular}

Tableau 2. Caractéristiques géométrique et mécanique de l'arbre 
Le formalisme mathématique utilisé pour étudier le comportement dynamique du rotor est l'équation de Lagrange:

$$
\frac{d}{d t}\left(\frac{\partial T}{\partial \dot{\bar{q}}_{i}}\right)-\frac{\partial T}{\partial \bar{q}_{i}}+\frac{\partial U}{\partial \bar{q}_{i}}+\frac{\partial \Re}{\partial \dot{\bar{q}}_{i}}=F_{d}
$$

Avec $F_{d}$ Fonction de dissipation, $U$ Energie potentielle, $T$ : Energie cinétique, $\mathfrak{R} \mathrm{La}$ fonction de dissipation et $\bar{q}_{i}$ :Vecteur coordonnées généralisées indépendantes.

\section{Généralités sur la méthode des éléments finis}

Dans le domaine de la mécanique des solides, la méthode des éléments finis est utilisée afin d'apporter la simplicité dans la modélisation et la résolution d'un problème [GOU 87]. Pour les structures simple et à faible degré de liberté, l'approche analytique est possible. Pour les structures complexes à plusieurs degrés de liberté, la mise en équation ainsi que la résolution sont fastidieuse, les méthodes numériques programmables s'imposent. Pour écrire les équations de la dynamique du rotor, nous avons utilisé l'approche de Lagrange.

A titre illustratif [BUC 95], considérons une poutre 3D (figure 11), soumise à diverse sollicitation. Cette poutre subit des contraintes qui impliquent des déformations qu'il faille de calculer.
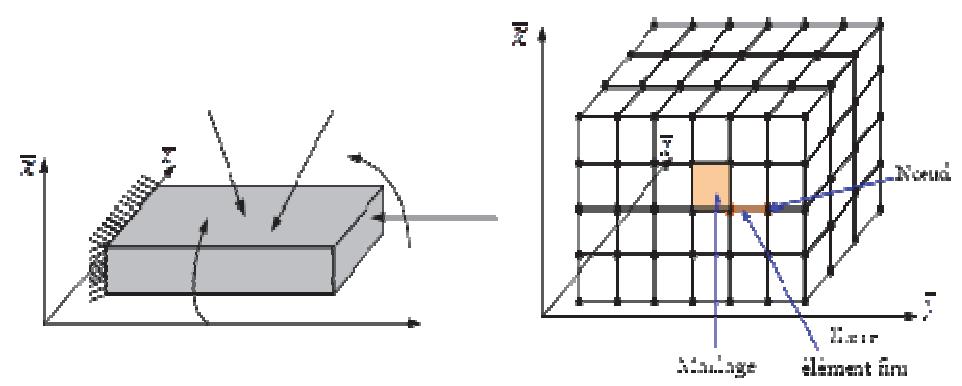

Figure 11. Eléments finis d'une poutre [BUC 95]

Le calcul est compliqué, il faut remplacer la structure continue par une autre en grillage. Il s'agit des barres dont les points de croisement s'appellent: «nœuds ». La structure sera calculée comme une structure discontinue discrétisée, constituée des petits éléments en forme géométriques triangulaires, curviligne ou rectangulaire. Le problème consiste de passer de la structure continue à une structure discrétisée équivalente. Isolons l'élément rectangulaire hachuré (figure 11), nous avons :

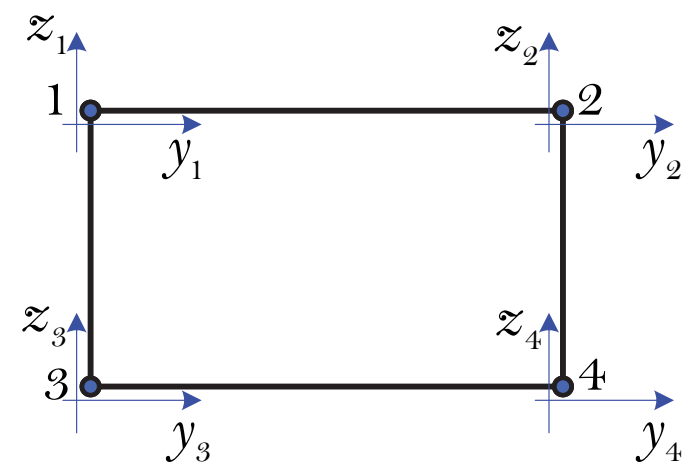

Figure 12. Elément fini de la poutre isolé [BUC 95] 
Nous voyons sur la figure 12 que sur chaque nœud, il y a des déplacements nodaux notés $y_{k}$ et $z_{k}$, cela conduit au champs de déplacement $\left\{\vec{g}_{e}\right\}$ et un système des forces généralisées noté $\left\{\vec{f}_{e}\right\}$, à chaque force correspond un déplacement :

$$
\left\{\vec{g}_{e}\right\}=\left[K^{e}\right]\left\{\vec{f}_{e}\right\}
$$

$\left[K^{e}\right]$ est la matrice de rigidité de l'élément fini

\subsection{Problème d'interpolation}

Au moyen de l'interpolation, il est possible de connaitre les déplacements en importe quel point à partir de deplacements noaux. Il existe des fonctions d'interpolation ou des fonctions de forme notées $\mathrm{N}_{\mathrm{i}}$ qui relient dans le cas de l'approche cinématique, les déplacements d'un point intérieur à un élément aux $\mathrm{n}$ déplacements nodaux $\mathrm{q}_{\mathrm{i}}$ qui sont les degrés de liberté. $v(y)$ est une combinaison linéaire des déplacements nodaux car c'est un déplacement d'un point quelconque de l'élément. Les coéfficients sont les valeurs des fonctions de forme en ce point :

$$
v(y)=\sum N_{i}(y) q_{i}
$$

Ces fonctions permettent le passage du problème continu au problème discret ; la reconstruction du champ de déplacement dans l'élément se fait à partir du déplacement des nœuds quelconques discrets.

La connexion entre élements obeï à la règle de continuité, sinon, on peut avoir des modes de déformation inacceptable du point de vue physique pour une structure continue. En ce qui concerne les problèmes d'elasticité, l'équation d'équilibre ne fait intervenir que la dérivée première des déformations (déplacments) : le champs de déplacement est de classe $\mathrm{C}^{0}$ pour chaque élément ; mais pour les problèmes de flexion, nous avons la dérivée seconde des déplacements pour l'équation d'équilibre; le deplacement est de classe $C^{1}$ pour chaque éléments. Nous avons sur la figure un problème d'interpolation.

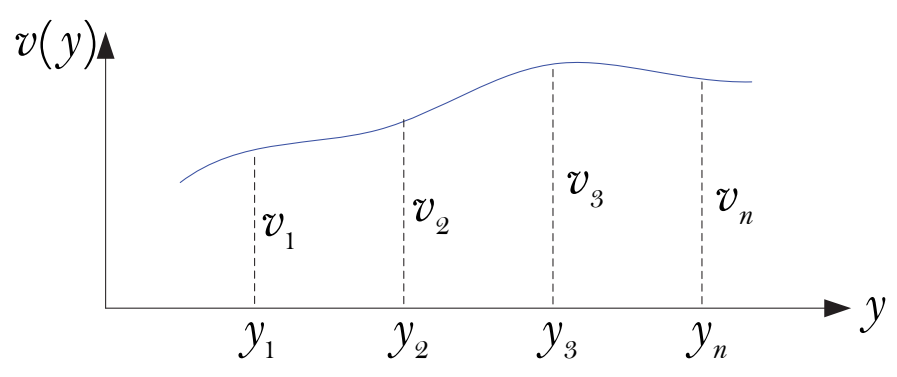

Figure 13. Illustration de l'interpolation d'une fonction

C'est l'interpolation de la fonction $v(y)$. Deux types d'interpolation existe: l'interpolation Hermitienne et l'interpolation Lagrangienne. La première est de classe $\mathrm{C}^{0}$ et la seconde de classe $\mathrm{C}^{1}$. La première sera utilisé dans la modélisation du rotor.

\subsection{Principe général de la méthode des éléments finis}

La méthode des élements finis consiste à chercher une solution approchée de la solution exacte sous la forme d'un champ d'un problème physique defini sur un domaine quelconque [PREU 12]. On procède de la manière suivante : la modélisation, la formulation du système d'équations différentielles et ses conditions aux limites, le maillage, le choix de la famille des champs locaux, la discrétisation, la 
résolution du problème discret, la construction des solutions approchées à partir des valeurs aux nœuds et la simulation ou la visualisation.

\section{Etablissement des éléments finis du rotor}

Il est question de déterminer les conditions d'équilibre du système, par la méthode énergétique, en l'occurrence l'énergie cinétique, de déformation et des fonctions de dissipation. Nous allons effectuer le calcul pour chaque élément du système.

\subsection{Modélisation élément fini du disque}

Le disque est modélisé par un nœud possédant quatre degrés de liberté: deux déplacements de rotation $\psi$ et $\theta$ autour des axes $0 \vec{x}$ et $0 \vec{z}$ et deux déplacements de translation $u$ et $w$ suivant les axes $0 \vec{x}$ et $0 \vec{z}$. Le disque est considéré comme rigide et symétrique.

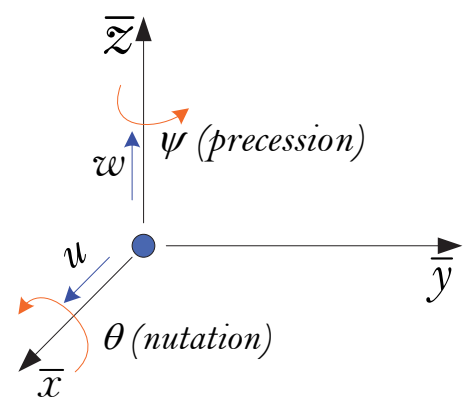

Figure14. Modèle élément fini du disque

Le champ de déplacements nodaux est donné par $\{\hat{q}\}=\left\{\begin{array}{l}u \\ w \\ \theta \\ \psi\end{array}\right\}$, l'énergie cinétique du disque est donnée par l'expression:

$$
T_{D}=\frac{1}{2} M_{D}\left[(\dot{u})^{2}+(\dot{w})^{2}\right]+\frac{1}{2} I_{D x}\left(\dot{\psi}^{2}+\dot{\theta}^{2}\right)+\frac{1}{2} I_{D y} \Omega^{2}+I_{D y}(\Omega \dot{\psi} \theta)
$$

Dans cette relation : le premier terme est l'énergie cinétique de translation du disque, le deuxième terme est l'énergie de rotation du disque autour des axes $0 \vec{x}$ et $0 \vec{z}$, le troisième terme est l'énergie de rotation du disque autour de son axe propre et le quatrième décrit l'éffet gyroscopique (ou de coriolis).

L'équation de Lagrange (2) appliquée à l'énergie cinétique du disque donne :

$$
\frac{d}{d t}\left(\frac{\partial T_{D}}{\partial \dot{q}}\right)-\frac{\partial T_{D}}{\partial q}=[M]\{\dot{q}\}+\Omega[I]\{\dot{q}\}
$$

Avec :

$$
M=\left[\begin{array}{cccc}
M_{D} & 0 & 0 & 0 \\
0 & M_{D} & 0 & 0 \\
0 & 0 & I_{D X} & 0 \\
0 & 0 & 0 & I_{D X}
\end{array}\right] I=\left[\begin{array}{cccc}
0 & 0 & 0 & 0 \\
0 & 0 & 0 & 0 \\
0 & 0 & 0 & -I_{D Y} \\
0 & 0 & I_{D Y} & 0
\end{array}\right] \quad q=\left\{\begin{array}{c}
u \\
w \\
\theta \\
\psi
\end{array}\right\}
$$




\subsection{Modélisation élément fini de l'arbre}

L'arbre est considéré comme une poutre de section constante et circulaire, le modèle élément fini est une barre. La barre est définie par ses deux extrémités qui constituent chacune un nœud. Un nœud possède trois déplacements de translation et trois de déplacement de rotation dans l'espace. Dans cette étude, nous ne considérons pas la translation suivant l'axe de rotation de l'arbre. Nous négligeons la rotation du nœud suivant l'axe de rotation de l'arbre. Donc chaque est représenté par deux déplacements de translation $u_{i}$ et $w_{i}$ suivants les axes $0 \vec{x}$ et $0 \vec{z}$, et deux déplacements de rotation $\psi_{i}$ et $\theta_{i}$ autour des axes $0 \vec{x}$ et $0 \vec{z}$.

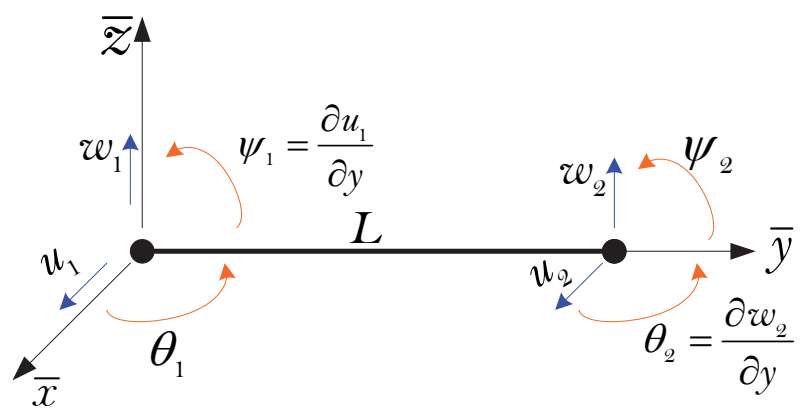

Figure 15. Modèle élément fini de l'arbre

Nous voyons que l'arbre possède 8 degrés de liberté. Le champ de déplacements nodaux peut alors s'écrire :

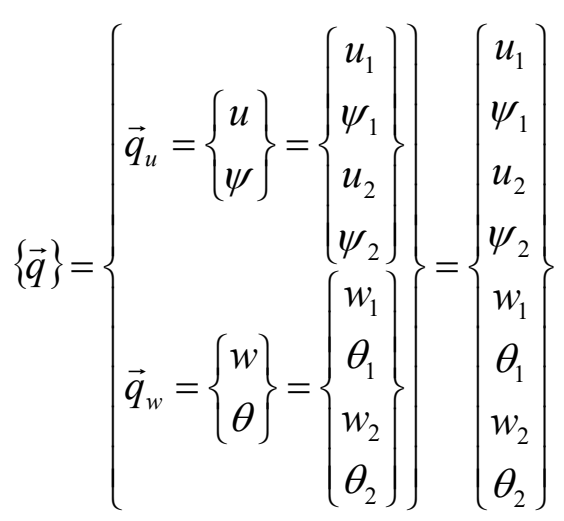

Le déplacement en un point de l'arbre est donné par :

$$
\left\{\begin{array}{l}
u(y) \\
w(y)
\end{array}\right\}=N(y)\left\{\begin{array}{l}
q_{u} \\
q_{w}
\end{array}\right\}
$$

$\mathrm{N}(\mathrm{y})$ est la fonction d'interpolation de Hermite

Pour un système à 8 degrés de liberté, la fonction d'interpolation de Hermite prend la forme matricielle suivante [GOU 87]:

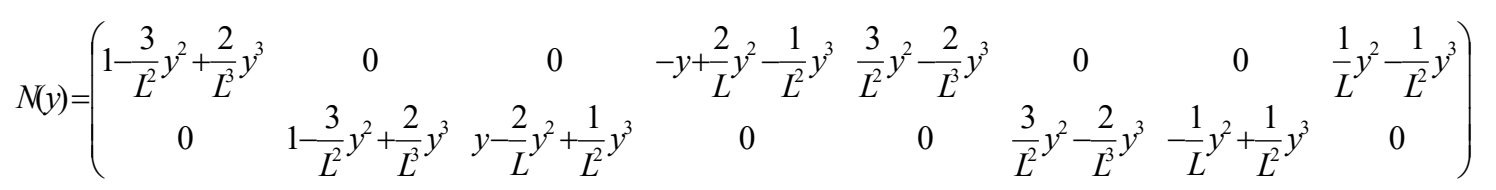

A partir des expressions du champ de déplacements, nous établissons l'énergie cinétique et l'énergie de déformation de l'arbre.

\subsubsection{Energie cinétique de l'arbre}

L'énergie cinétique de l'arbre est donnée par : 


$$
T=\frac{1}{2} \rho S_{a} \int_{0}^{L}\left[(\dot{u})^{2}+(\dot{w})^{2}\right] d y+\frac{1}{2} \rho I \int_{0}^{L}\left(\dot{\psi}^{2}+\dot{\theta}^{2}\right) d y+\rho I \Omega^{2} L+2 \rho I_{D y} \Omega \int_{0}^{L}(\dot{\psi} \theta) d y
$$

Dans la relation (12), il y a quatre termes : le premier terme est l'énergie cinétique d'une poutre en flexion (énergie de translation), le deuxième terme indique l'énergie de rotation, le troisième terme est constant et le dernier terme est dû à l'effet gyroscopique.

Sachant que $u=N_{1}(y)\left\{q_{u}\right\}, w=N_{2}(y)\left\{q_{w}\right\}, \dot{u}=N_{1}(y)\left\{\dot{q}_{u}\right\}, \dot{w}=N_{2}(y)\left\{\dot{q}_{w}\right\} \psi=-\frac{\partial N_{1}(y)}{\partial y}\left\{q_{u}\right\}$, $\theta=\frac{\partial N_{2}(y)}{\partial y}\left\{q_{w}\right\}, \dot{\psi}=-\frac{\partial N_{1}(y)}{\partial y}\left\{\dot{q}_{u}\right\}$ et $\dot{\theta}=\frac{\partial N_{2}(y)}{\partial y}\left\{\dot{q}_{w}\right\}$

L'énergie cinétique devient :

$$
\begin{aligned}
T_{a}= & \frac{1}{2} \rho S_{a} \int_{0}^{L}\left[\left(N_{1}(y)\left\{\dot{q}_{u}\right\}\right)^{2}+\left(N_{2}(y)\left\{\dot{q}_{w}\right\}\right)^{2}\right] d y+\frac{1}{2} \rho I \int_{0}^{L}\left[\left(\frac{\partial \mathrm{N}_{1}(y)}{\partial y}\left\{\dot{q}_{u}\right\}\right)^{2}+\left(\frac{\partial \mathrm{N}_{2}(y)}{\partial y}\left\{\dot{q}_{w}\right\}\right)^{2}\right] d y \\
& +\rho I \Omega^{2} L+2 \rho I_{D y} \Omega \int_{0}^{L}\left[\left(\frac{\partial \mathrm{N}_{1}(y)}{\partial y}\left\{\dot{q}_{u}\right\}\right) \cdot\left(\frac{\partial \mathrm{N}_{2}(y)}{\partial y}\left\{q_{w}\right\}\right)\right] d y
\end{aligned}
$$

Sous la forme quadratique, l'expression peut encore s'écrire :

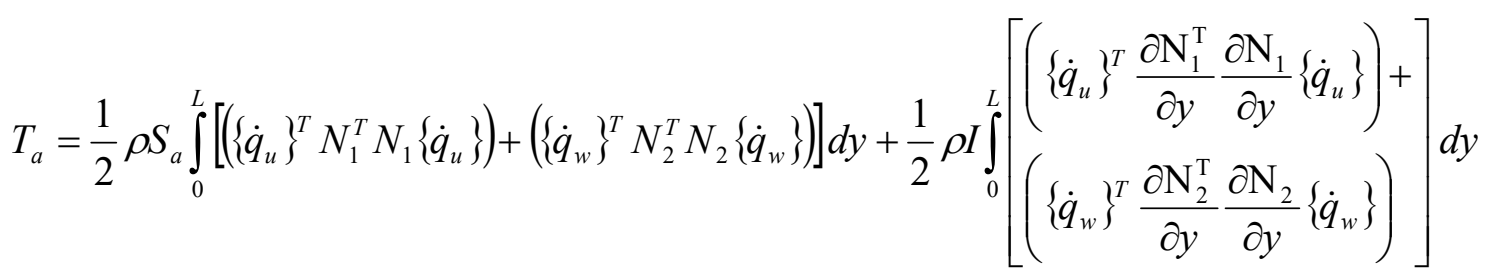

$$
\begin{aligned}
& +\rho I \Omega^{2} L+2 \rho I_{D y} \Omega \int_{0}^{L}\left\{\dot{q}_{u}\right\}^{T} \frac{\partial \mathrm{N}_{1}^{\mathrm{T}}}{\partial y} \frac{\partial \mathrm{N}_{2}}{\partial y}\left\{q_{w}\right\} d y
\end{aligned}
$$

Posons $\quad M_{1}=\rho S_{a} \int_{0}^{L} N_{1}^{T} N_{1} d y \quad$ et $\quad M_{2}=\rho S_{a} \int_{0}^{L} N_{2}^{T} N_{2} d y, \quad I_{1}=\rho I \int_{0}^{L} \frac{\partial N_{1}^{T}}{\partial y} \frac{\partial N_{1}}{\partial y} d y \quad$ et $\quad I_{2}=\rho I \int_{0}^{L} \frac{\partial N_{2}^{T}}{\partial y} \frac{\partial N_{2}}{\partial y} d y \quad$ et $G=\rho I_{D y} \int_{O}^{L} \frac{\partial N_{1}^{T}}{\partial y} \frac{\partial N_{2}}{\partial y} d y$ respectivement matrices masse, effet d'inertie de rotation de la section de l'arbre et effet gyroscopique.

L'expression de l'énergie peut s'écrire :

$$
T_{a}=\frac{1}{2}\left(\left\{\dot{q}_{u}\right\}^{T} M_{1}\left\{\dot{q}_{u}\right\}\right)+\frac{1}{2}\left(\left\{\dot{q}_{w}\right\}^{T} M_{2}\left\{\dot{q}_{w}\right\}\right)+\frac{1}{2}\left(\left\{\dot{q}_{u}\right\}^{T} I_{1}\left\{\dot{q}_{u}\right\}\right)+\frac{1}{2}\left(\left\{\dot{q}_{w}\right\}^{T} I_{2}\left\{\dot{q}_{w}\right\}\right)+\rho I \Omega^{2} L+\left(\left\{\dot{q}_{u}\right\}^{T} G\left\{q_{w}\right\}\right) \Omega
$$

En appliquant l'équation de Lagrange (2) à la relation (15), on obtient :

$$
\frac{d}{d t}\left(\frac{\partial T_{a}}{\partial\{\dot{q}\}}\right)-\frac{\partial T_{a}}{\partial\{q\}}=\left[\left(M+M_{S}\right)\right]\{\ddot{q}\}+[C]\{\dot{q}\}
$$

$M$ est matrice provenant des matrices masses, $M_{s}$ vient des matrices dues à l'effet de rotation et $C$ la matrice liée à l'effet gyroscopique. Les matrices $\mathrm{M}, \mathrm{M}_{\mathrm{S}}$ et $\mathrm{C}$ sont des matrices carrées symétriques $8 \times 8$, elles sont données par : 


$$
\begin{aligned}
& M=\frac{\rho S_{a}}{420} L \cdot\left[\begin{array}{cccccccc}
156 & 0 & 0 & -22 L & 54 & 0 & 0 & 13 L \\
& 156 & 22 L & 0 & 0 & 54 & -13 L & 0 \\
& & 4 L^{2} & 0 & 0 & 13 L & -3 L^{2} & 0 \\
& & & 4 L^{2} & -13 L & 0 & 0 & -3 L^{2} \\
& & & & 156 & 0 & 0 & 22 L^{2} \\
& & I d e m & & & 156 & -22 L & 0 \\
& & & & & & 4 L^{2} & 0 \\
& & & & & & & 4 L^{2}
\end{array}\right]
\end{aligned}
$$

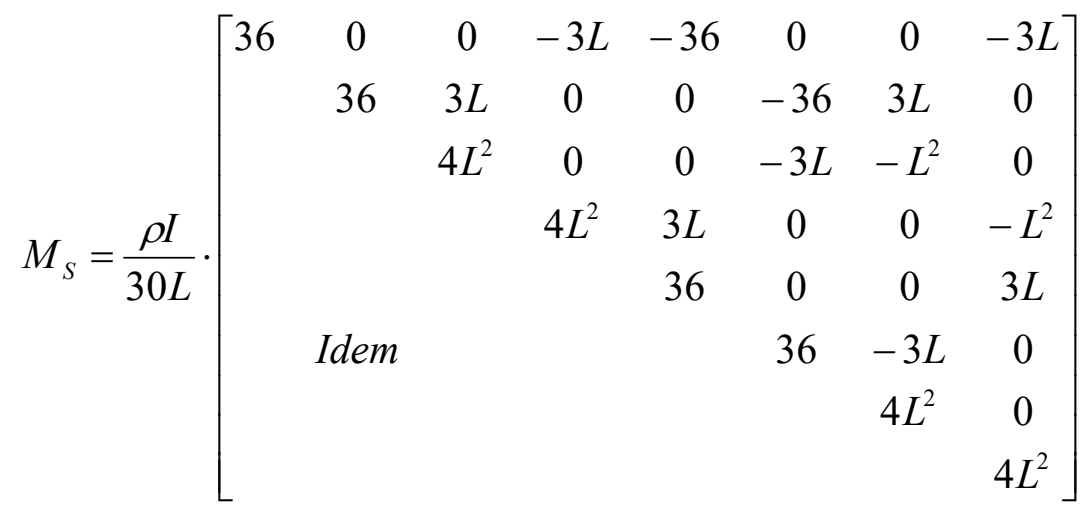

$$
\begin{aligned}
& C=\frac{\rho \Omega I_{D y}}{15 L} \cdot\left[\begin{array}{cccccccc}
0 & -36 & -3 L & 0 & 0 & 36 & -3 L & 0 \\
& 0 & 0 & -3 L & -36 & 0 & 0 & -3 L \\
& & 0 & -4 L^{2} & -3 L & 0 & 0 & L^{2} \\
& & & 0 & 0 & -3 L & -L^{2} & 0 \\
& & & & 0 & -36 & 3 L & 0 \\
& \text { Anti- } & \text { sym } & & & 0 & 0 & 3 L \\
& & & & & & 0 & -4 L^{2} \\
& & & & & & & 0
\end{array}\right]
\end{aligned}
$$

\subsubsection{Energie de déformation de l'arbre}

L’énergie de déformation du rotor est donnée par :

$$
U_{a}=\frac{E I}{2} \int_{0}^{L}\left[\left(\frac{\partial^{2} w}{\partial y^{2}}\right)^{2}+\left(\frac{\partial^{2} u}{\partial y^{2}}\right)^{2}\right] d y
$$

C'est l'expression de l'énergie de déformation d'un rotor symétrique soumis en flexion dans le repère fixe $(\vec{x} 0 \vec{z})$.

Sachant que $u=N_{1}(y) q_{u}, \frac{\partial^{2} u}{\partial y^{2}}=\frac{\partial^{2} N_{1}(y)}{\partial y^{2}} q_{u}, w=N_{2}(y) q_{w}$ et $\frac{\partial^{2} w}{\partial y^{2}}=\frac{\partial^{2} N_{2}(y)}{\partial y^{2}} q_{w}$ nous obtenons :

$$
U_{a}=\frac{E I}{2} \int_{0}^{L}\left[\left(\frac{\partial^{2} N_{1}(y)}{\partial y^{2}} q_{u}\right)^{2}+\left(\frac{\partial^{2} N_{2}(y)}{\partial y^{2}} q_{w}\right)^{2}\right] d y
$$

Sous forme quadratique on peut encore écrire :

$$
U_{a}=\frac{E I}{2} \int_{0}^{L}\left[\left(q_{u}^{T} \frac{\partial^{2} N_{1}^{T}(y)}{\partial y^{2}} \frac{\partial^{2} N_{1}(y)}{\partial y^{2}} q_{u}\right)+\left(q_{w}^{T} \frac{\partial^{2} N_{2}^{T}(y)}{\partial y^{2}} \frac{\partial^{2} N_{2}(y)}{\partial y^{2}} q_{w}\right)\right] d y
$$

En posant $K_{1}=E I \int_{0}^{L} \frac{\partial^{2} N_{1}^{T}(y)}{\partial y^{2}} \frac{\partial^{2} N_{1}(y)}{\partial y^{2}} d y$ et $K_{2}=E I \int_{0}^{L} \frac{\partial^{2} N_{2}^{T}(y)}{\partial y^{2}} \frac{\partial^{2} N_{2}(y)}{\partial y^{2}} d y$; ces sont les matrices de raideur de l'arbre, l'énergie de déformation de l'arbre devient : 


$$
U_{a}=\frac{1}{2}\left(q_{u}^{T} K_{1} q_{u}\right)+\frac{1}{2}\left(q_{w}^{T} K_{2} q_{w}\right)
$$

Après application de l'équation de Lagrange (2) à la relation (23), nous avons :

$$
\frac{\partial U_{a}}{\partial\{q\}}=K\{q\}
$$

$K$ est définie comme ci-dessous :

$$
K=\frac{E I}{L^{3}} \cdot\left[\begin{array}{cccccccc}
12 & 0 & 0 & -6 L & -12 & 0 & 0 & -6 L \\
& 12 & 6 L & 0 & 0 & -12 & 6 L & 0 \\
& & 4 L^{2} & 0 & 0 & -6 L & 2 L^{2} & 0 \\
& & & 4 L^{2} & 6 L & 0 & 0 & 2 L^{2} \\
& & & & 12 & 0 & 0 & 6 L \\
& & & & & \\
& & & & & 12 & -6 L & 0 \\
& & & & & & 4 L^{2} & 0 \\
& & & & & & & 4 L^{2}
\end{array}\right]
$$

\subsection{Modélisation de balourd}

L'énergie cinétique de balourd $\mathrm{T}_{\mathrm{B}}$ est donnée par :

$$
T_{B}=m_{B} \Omega d\{(\dot{u} \cos \Omega t)-(\dot{w} \sin \Omega t)\}
$$

Après application des équations de Lagrange (2) à la relation (26), on obtient :

$$
\frac{d}{d t}\left(\frac{\partial T_{B}}{\partial \dot{q}}\right)-\frac{\partial T_{B}}{\partial q}=-m_{B} \Omega^{2} d\left\{\begin{array}{l}
\sin (\Omega t) \\
\cos (\Omega t)
\end{array}\right\}
$$

Avec $q=\left\{\begin{array}{l}u \\ w\end{array}\right\}$

Les balourds sont les forces d'excitations asynchrones.

\subsection{Modélisation de paliers}

Les paliers sont modélisés en considérant l'arbre comme un solide viscoélastique linéaire (figure 6), il s'agit du modèle de Kelvin, caractérisé par un amortissement $C$ en parallèle suivant $(0 \vec{x})$ et $(0 \vec{z})$ et une raideur $\mathrm{k}$ [TAL 07].
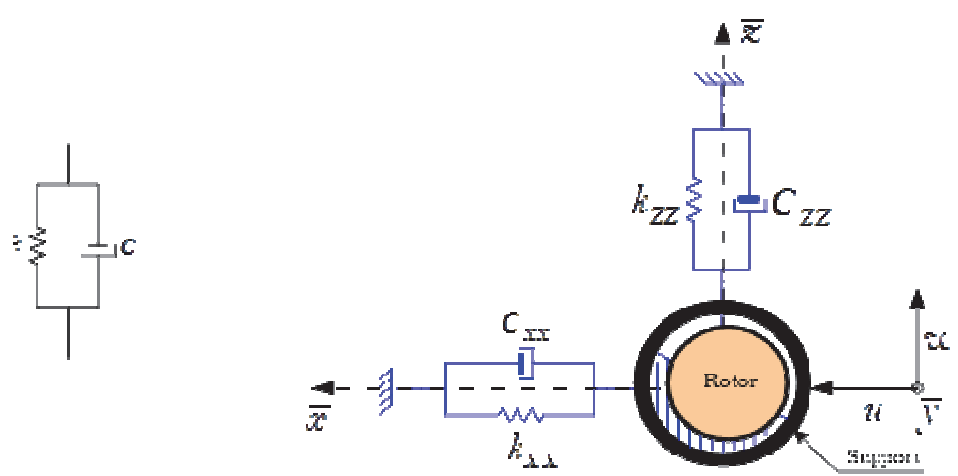

Figure 16. Comportement viscoélastique de l'arbre dans les paliers

La relation des forces dissipatives est donnée par : 


$$
\left(\begin{array}{l}
F_{u} \\
F_{\psi} \\
F_{w} \\
F_{\theta}
\end{array}\right)=-\left(\begin{array}{cccc}
k_{X X} & 0 & k_{X Z} & 0 \\
0 & 0 & 0 & 0 \\
k_{Z X} & 0 & k_{Z Z} & 0 \\
0 & 0 & 0 & 0
\end{array}\right) \cdot\left(\begin{array}{c}
u \\
\psi \\
w \\
\theta
\end{array}\right)-\left(\begin{array}{cccc}
C_{X X} & 0 & C_{X Z} & 0 \\
0 & 0 & 0 & 0 \\
C_{Z X} & 0 & C_{Z Z} & 0 \\
0 & 0 & 0 & 0
\end{array}\right) \cdot\left(\begin{array}{c}
\dot{u} \\
\dot{\psi} \\
\dot{w} \\
\dot{\theta}
\end{array}\right)
$$

\section{Modélisation du rotor}

La modélisation mathématique du mouvement du rotor se fait en utilisant les matrices élémentaires obtenues au point 3 . Nous obtenons une équation de la forme :

$$
[M]\{\ddot{q}\}+[C(\Omega)]\{\dot{q}\}+[K]\{q\}=F_{\text {totales }}
$$

Avec $[M]$ la matrice masse, $\{q\}$ le champ de déplacements nodaux, $[C(\Omega)]$ une matrice non symétrique dépendant de la vitesse de rotation du système et tient compte de l'effet gyroscopique, $[K]$ la matrice de raideur caractéristique des paliers et $F_{\text {totales }}$ la somme de forces excitatrices du rotor.

\section{Simulation numérique du mouvement du rotor}

Afin de déterminer les caractéristiques modales du rotor, il sera question de résoudre l'équation (30).

$$
[M]\{\ddot{q}\}+[C(\Omega)]\{\dot{q}\}+[K]\{q\}=0
$$

A partir cette équation (30), nous avons : les pulsations propres, les valeurs propres, les vecteurs propres, les coordonnées modales ainsi que la matrice modale du rotor. Le nombre de degré de liberté est élevé, la résolution manuelle est fastidieuse ; l'utilisation des méthodes itératives et programmables devient inévitables. Le logiciel nous permettra de déduire le comportement global du rotor et de tirer certaines informations importantes sur le comportement dynamique du rotor. Le logiciel simule l'équation (29). Il s'agit de l'influence du balourd sur les paliers et sur le niveau des amplitudes des vibrations.

Il existe plusieurs logiciels d'étude et de prévision du comportement dynamique du rotor: le Sysrotor, le Rotorinsa, Matlab, Ansys, etc. pour la caractérisation du comportement dynamique du rotor, nous avons utilisé le logiciel Rotorinsa 4.06. Ce logiciel est basé sur la méthode des éléments finis.

\subsection{Modélisation sous Rotorinsa du rotor}

\subsubsection{Modèle géométrique et éléments finis du rotor}

Il s'agit d'une mise en place de chaque élément du rotor sur le nœud correspondant. 5 éléments d'arbres chacun modélisé par une barre avec 2 nœuds à ses extrémités, 2 disques placés chacun modélisé par un nœud et placé respectivement aux nœuds 3 et 4, 2 paliers placés respectivement aux nœuds 2 et 5 . Il n'y a pas d'accouplement, ni d'éléments supplémentaires, pas d'attraction magnétique, ni de paliers magnétiques actifs dans ce modèle du rotor. 


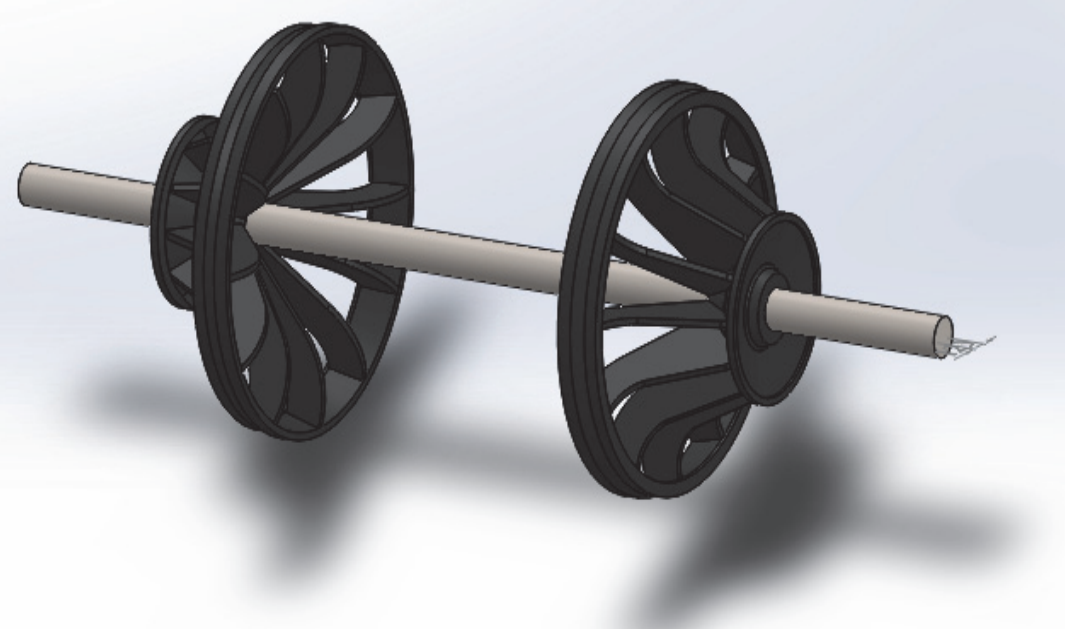

Figure 17. Rotor

Sur la figure 17. , nous avons le rotor faisant objet de notre étude, c'est dessin est effectué sur le logiciel solidword, en respectant les différentes dimensions. Après avoir inséré les données du rotor (Tableau 1 et 2) dans Rotorinsa, nous obtenons la figure 18. Le maillage est fait en 7 éléments de barre.

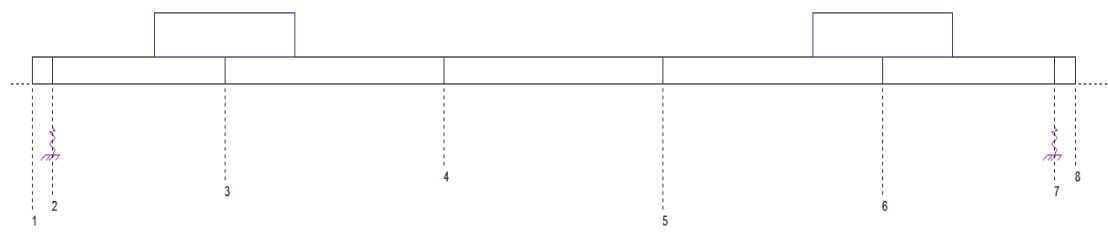

Figure 18. Modèle éléments finis du Rotor

\subsubsection{Résultats}

\subsubsection{Modes propres en rotation}

Ces modes propres permettent d'analyser les déplacements nodaux suivants les différents axes et les contraintes y engendrées par les modes des déformations. Le tableau 3 résume les facteurs d'amortissement à chaque mode et les fréquences propres correspondantes :

\begin{tabular}{|c|c|c|c|c|}
\hline $\begin{array}{l}\text { Nombre de } \\
\text { Modes }\end{array}$ & 1 & 2 & 3 & 4 \\
\hline $\begin{array}{c}\text { Facteur } \\
\text { d'amortissement }\end{array}$ & $4,1.10^{-5}$ & $1,11.10^{-5}$ & $2,62.10^{-4}$ & $9,82.10^{-8}$ \\
\hline $\begin{array}{c}\text { Fréquences } \\
\text { propres (Hz) }\end{array}$ & 8,07 & 20,97 & 21,08 & 40,05 \\
\hline
\end{tabular}

Tableau 3. Facteur d'amortissement 
Les figures 19, 20, 21 et 22 ci-dessous donnent les allures de ces modes propres en rotation pour le système tournant à une vitesse de rotation de $250 \mathrm{tr} / \mathrm{min}$.

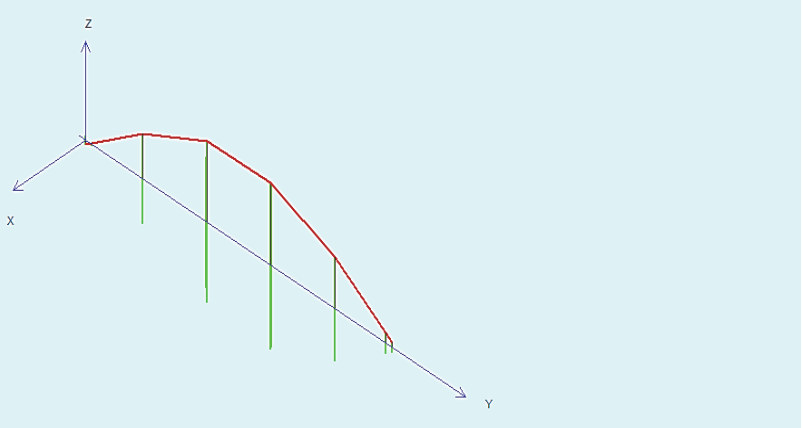

Figure 19. $1^{\text {er }}$ Mode propre en rotation de précession inverse

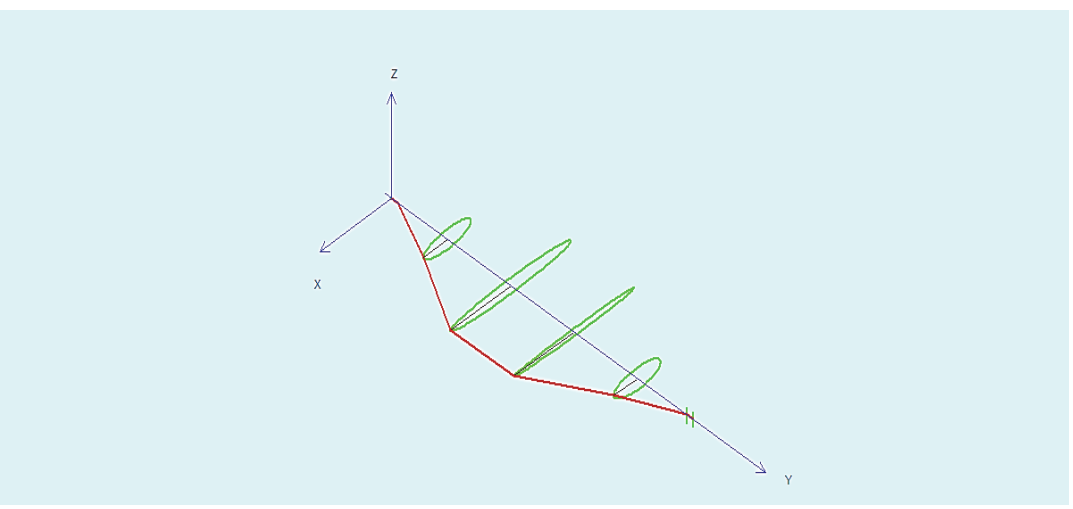

Figure 20. $2^{\text {ème }}$ Mode propre en rotation de précession inverse

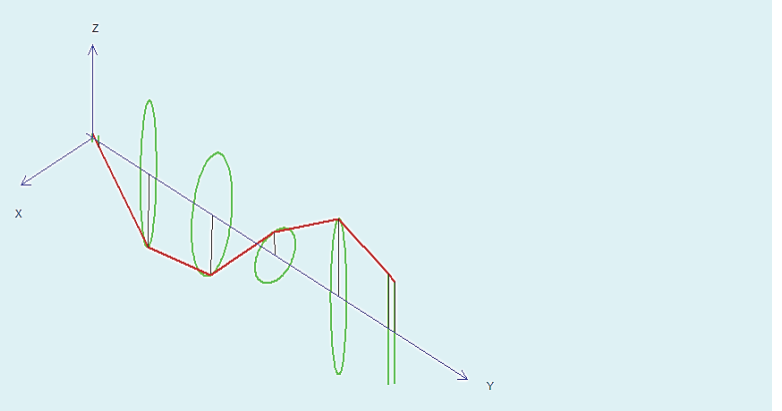

Figure 21. $3^{\text {ème }}$ Mode propre en rotation

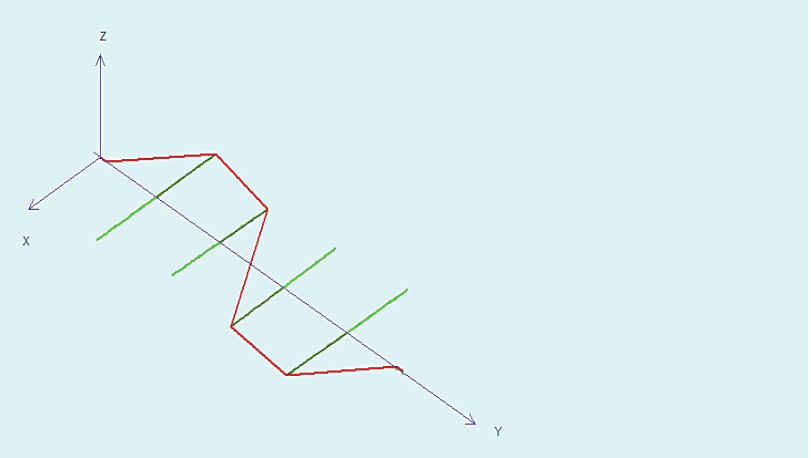

Figure 22. $4^{\text {ème }}$ Mode propre en rotation 


\subsubsection{Déformées sous excitation de balourd}

Les déformées ci-dessous sont obtenues pour un balourd de $3.10^{-2} \mathrm{~kg}$ appliqué sur chaque disque. Au premier cas, nous avons considéré une vitesse de rotation de $20 \mathrm{tr} / \mathrm{min}$, suivies de 250 et de 530 $\mathrm{tr} / \mathrm{min}$. à chaque réponse nous avons les résultats inscrits dans le tableau suivant .

\begin{tabular}{|l|c|c|c|}
\hline $\begin{array}{l}\text { Vitesse de rotation } \\
(\text { tr/min })\end{array}$ & 200 & 250 & 530 \\
\hline Amplitude max $(\boldsymbol{m})$ & $8,9.10^{-7}$ & $1,57 \cdot 10^{-6}$ & $2,47.10^{-5}$ \\
\hline Fréquence propre $(\boldsymbol{H} \mathbf{z})$ & 3,33 & 4,17 & 8,83 \\
\hline
\end{tabular}

Tableau 4. Amplitude des vibrations

Figure 23. Déformée sous balourd à $200 \mathrm{tr} / \mathrm{min}$

Figure 24. Déformée sous balourd à $250 \mathrm{tr} / \mathrm{min}$

Figure 25. Déformée sous balourd à $530 \mathrm{tr} / \mathrm{min}$ 
Remarquons, de ce qui précède sur les figures 23,24 et 25 , plus la vitesse de rotation est élevée, plus l'effet de balourd devient prépondérant. Et par conséquent les déplacements étant nuls aux paliers, ces derniers deviennent donc très sollicités par des forces dynamiques et susceptibles de connaitre l'usure des matériaux anti friction; et enfin un déséquilibrage de l'arbre. Les amplitudes des vibrations indiquées dans le tableau 4 sont inadmissibles selon la norme ISO 10816-3.

\subsubsection{Réponse à une excitation de balourd asynchrone}

Sur la figure (26) nous remarquons, pour un balourd minimal de $3.10^{-2} \mathrm{~kg}$, la présence d'un pic de résonnance d'amplitude variant entre $10^{-5} \mathrm{~m}$ et $0,009 \mathrm{~m}$ à $483 \mathrm{tr} / \mathrm{min}$. ces amplitudes sont plus importantes aux nœuds 3, 4, 5 et 6 tandis qu'elles sont moindres aux nœuds 1 et 2 . Donc les amplitudes de vibration sont prépondérantes de la première roue de gauche vers la droite du rotor.

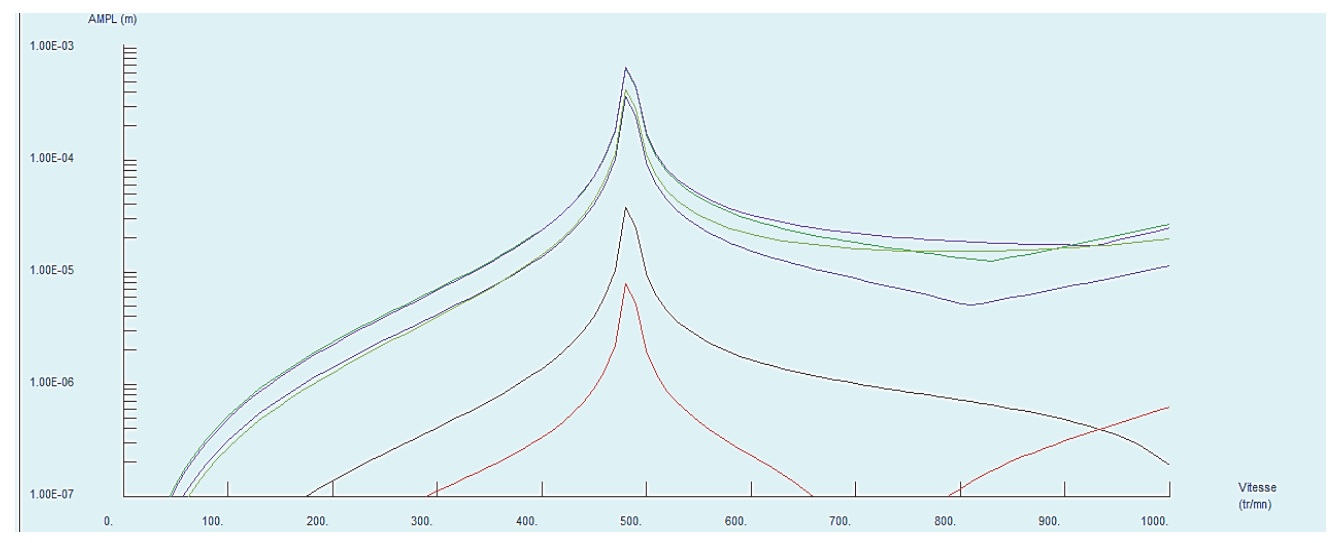

Figure 26. Réponse sous excitation asynchrone

\section{Conclusion}

Cet article présente une démarche pour comprendre les phénomènes vibratoires et les bruits sur un rotor, un cas typique d'un rotor d'une centrale hydraulique est considéré. Ce travail montre la complicité d'une telle étude. Pour un cas industriel, la mise en équation du mouvement du rotor par la méthode des éléments finis est ardue, le logiciel Rotorinsa a permis de simuler l'influence du balourd sur le comportement vibratoire du rotor. A cet effet, les amplitudes des vibrations sont élevées lorsque la vitesse du rotor augmente et sont inadmissible selon la norme ISO 10816-3. Une étude sur l'équilibrage du rotor en utilisant la méthode des coefficients d'influence peut être abordé afin d'atténuer les vibrations et les bruits.

\section{Bibliographie}

[BRA 10] BRAHMI H., Etude du comportement vibratoire et simulation numérique des charges dynamiques d'un rotor flexible, Université M'Hamed BOUGARA, Alger, 2010.

[CHE 08] CHELLIL A., Identification et modélisation par éléments finis des charges dynamiques du rotor principal d'hélicoptère, Université M'Hamed BOUGARA, Alger, 2008.

[SAI 13] SAIMI A., Comportement vibratoire des roues aubagées, Université Aboubeker Belkaid-Tlemcen, Alger, 2013.

[GHA 14] GHABBI R., Analyse des phénomènes vibratoires en dynamique des rotors - modélisation et expérimentation, Université de BATNA, Alger, 2014.

[LAL 96] LALANNE M. et FERRARIS G., Dynamique des rotors en flexion, Technique d'ingénieur, Nº serie b5110, 1996.

[STE 96] STEFAN P. et IORDANOFF Y., Butées et paliers aérodynamiques, Technique d'ingénieur, $\mathrm{N}^{\circ}$ serie b5335, 1996.

[BER 84] BERNASCONI O., Vibrations de torsion des arbres de machines - calcul d'un modèle à paramètres repartis discontinus, Ecole polytechnique fédérale de Lausanne, 1984. 
[TAL 07] TALA IGHIL N., Contribution à l'étude des paliers en régime hydrodynamique instationnaire - influence des états de surface, Université des sciences de technologie d'Oran, 2007.

[FAV 98] LAKEHAL A., Prédiction d'avaries et maîtrise du comportement dynamique des machines tournantes, Université de BADJI MOKHTAR-ANNABA, 2013.

[ROQ 07] ROQUES S., Modélisation du comportement dynamique couplé rotor - stator d'une turbine en situation accidentelle, Université de Nantes, 2007.

[LEM 03] DEMAILLY D, Etude du comportement non linéaire dans le domaine fréquentiel - Application à la dynamique rotor, Ecole centrale de Lyon, 2003.

[PRE 12] PREUMONT A., Twelve lectures on structural dynamics, Université libre de Bruxelles, 2012.

[MON 14b] CRAVEUR J., Modélisation des éléments finis, Dunod, 2008.

[BUC 95] BUCHANAN G., Finite element analysis - theory an problems, McGRAW - HILL, 1995

[GOU 87] GOURI D. et GILBERT T., une présentation de la méthode des éléments finis, collection université de compiegne, Paris, 1987.

[JEN 05] JENNY M., Machines à fluides - turbomachines, Mines Nancy, 2014.

[GER 05] GERLEMENT G. et DATOUSSAID S., Complément de résistance des matériaux - Théorie, FPMS, 2005.

[REG 05] REGGIO M. et TREPANIER JY., Théorie des turbomachines, Ecole polytechnique Montreal, 2005.

[GOJ 05] GOJON R., Critères de choix d'un palier, Technique d'ingénieur, N Serie b 5300, 1999.

[PUG 03] PUGNET JM., Equilibrage de rotors rigides, Technique d'ingénieur, N Serie Bm 5130, 2003. 